\title{
Management of bleeding with non vitamin K oral anticoagulants
}

\begin{abstract}
Bleeding is a potential side effect with the use of anticoagulation. The non-vitamin $\mathrm{K}$ oral anticoagulants (NOACS) are as effective as warfarin for stroke prevention in non-valvular atrial fibrillation and also for the prevention and treatment of venous thromboembolic disease. They have demonstrated favourable safety profiles when compared to warfarin with significantly lower risk of haemorrhagic stroke. Furthermore regular therapeutic monitoring is not necessary which may be more acceptable to patients, leading to its widespread use. However if major bleeding occurs there is no universal index of measure for the coagulation effect of the NOACS or antidotes available. In patients who require invasive procedures guidance has been given with regards to drug discontinuation. The management of bleeding sequelae from NOACS may be less familiar to non-specialists and this article sets out to review the current literature regarding bleeding management of the four NOACS.
\end{abstract}

Volume 7 Issue I - 2016

\author{
Shabnam Rashid,' Stephanie Hughes ${ }^{2}$ \\ 'Leeds General Infirmary, United Kingdom \\ ${ }^{2}$ Manchester Royal Infirmary, United Kingdom
}

Correspondence: Shabnam Rashid, Leeds General Infirmary, Great George Street, Leeds, West Yorkshire, LSI 3EX, United Kingdom, Tel 0113 2432799, Fax 0113 392575I, Email shabnamrashid@doctors.org.uk

Received: September 20, 2016 | Published: November 08, 2016
Abbreviations: NOACS, non vitamin K oral anticoagulants; $\mathrm{AF}$, atrial fibrillation; APTT, activated partial thromboplastin time; $\mathrm{TT}$, thrombin time; $\mathrm{CrCl}$, creatinine clearance; $\mathrm{PCC}$, prothrombin complex

\section{Introduction}

The non-vitamin K oral anticoagulants (NOACS) are commonly prescribed as therapeutic alternatives to warfarin. The most important potential adverse effect with anticoagulant use is bleeding. The management of warfarin induced coagulopathy is well established. The degree of international normalised ratio (INR) elevation along with bleeding severity is used to guide treatment such as the use of vitamin K to reverse warfarin's effect on the coagulation cascade. The management of bleeding with the NOACS is unclear as there is no universal reversal agent. At present the effects of dabigatran can be reversed by the use of idarucizumab whilst the remaining NOACS do not have approved antidotes. In the case of bleeding the first stage is to stop the NOAC, support the circulation with intravenous fluids and transfusing red blood cells if necessary. Other measures include prescribing prothrombin complex, tranexamic acid, activated charcoal and recombinant activated factor VIIa depending on the degree of bleeding. This article will consider how factors such as drug half-life, patient renal function and laboratory coagulation tests can help guide management decisions in patients who encounter bleeding with the NOACS or require invasive surgical procedures necessitating drug discontinuation.

The non vitamin $\mathrm{K}$ oral anticoagulants (NOACS) include dabigatran a direct thrombin inhibitor, rivaroxaban, edoxaban and apixaban which are direct factor Xa inhibitors. These are indicated in patients with non valvular atrial fibrillation (AF) as well as prevention and treatment of deep vein thrombosis and pulmonary embolus. They are commonly prescribed as there is no requirement for monitoring of coagulation profile or frequent dose adjustments unlike the vitamin $\mathrm{K}$ antagonists. Furthermore no specific dietary restrictions are necessary with the NOACS.

The incidence of major bleeding with warfain such as intracranial, gastrointestinal, genitourinary and respiratory is $1-3 \%$ per patient year. ${ }^{1-3}$ Compared with warfarin, there is a lower risk of intracranial hemorrhage with the NOACS. Apixaban and Dabigatran (110mg twice daily) demonstrated a lower risk of major bleeding from any site. $^{4-6}$ Rivaroxaban and Dabigatran (150mg tiwce daily) had an increased risk of gastrointestinal bleeding compared to warfarin. Apixaban was associated with fewer gastrointestinal bleeding events when compared to warfarin, but this was not statistically significant. ${ }^{7}$ In a recent meta-analysis, apixaban demonstrated lower rates of both major bleeding and gastrointestinal bleeding when compared to dabigatran. There are no randomized trial data with head to head comparison of the NOACS. ${ }^{8}$

The clearance of NOACS is rapid and occurs gradually over 12-24hours if renal and liver function are within normal range. If bleeding is encountered with warfarin the anticoagulant effect can be reversed with fresh frozen plasma and vitamin k. Protamine sulfate is recommended for the reversal of unfractionated heparin and low molecular weight heparin. Recently idarucizumab has been approved and licensed for the reversal of the anticoagulant effect of dabigatran. There are no specific reversal agents for rivaroxaban, apixaban, and edoxaban. Therefore, when prescribing such agents the risk of bleeding needs to be taken into consideration.

Laboratory coagulation tests are not reliable to measure the anticoagulant effect of NOACs but they do provide some guidance with regards to plasma levels of the NOACS. Laboratory testing maybe required in certain situations where there is significant bleeding or in patients who require urgent surgery where the presence of the anticoagulant effect of NOAC may hinder surgery. ${ }^{8}$

\section{Dabigatran and monitoring of coagulation}

Dabigatran was the first NOAC to be approved. It is available in two doses; $110 \mathrm{mg}$ BD or $150 \mathrm{mg}$ BD with the onset of action between 1-3hours. A normal activated partial thromboplastin time (APTT) excludes the anticoagulant effect of dabigatran. An Increased APTT, thrombin time (TT), and ecarin clotting time (ECT) an assay to measure thrombin generation suggest clinical relevant levels of the drug in circulation. There is a risk of bleeding if the APTT is $>2 x$ the upper limit of normal, TT $>200 \mathrm{ng} / \mathrm{ml}$ or $>65$ seconds or ECT $>3 \mathrm{x}$ upper limit of normal. ${ }^{9}$ The TT is most sensitive test to assess for the anticoagulant effect of dabigatran however, the APTT is readily available in most laboratories. ${ }^{8,10}$ The prothrombin time (PT) is relatively insensitive to dabigatran and antifactor Xa levels are not useful. ${ }^{11}$ 
In the RE-LY trial, dabigatran and warfarin were compared in patients with non valvular AF. Although dabigatran reduced embolic complications there was a significant increase in gastrointestinal bleeding likely to be caused by the presence of the active drug in the gastrointestinal tract promoting bleeding through a topical effect. ${ }^{5}$

\section{Dabigatran and renal function}

The half-life of dabigatran is between12 - 17hours in patients with normal renal function. In patients with a high risk of bleeding drug discontinuation should occur 1-2days before the procedure. The drug is excreted predominantly via the kidneys therefore in patients who have impaired kidney function then discontinuation of dabigatran is required earlier. With a Creatinine Clearance $(\mathrm{CrCl})$ of $30-49 \mathrm{~mL} /$ min, dabigatran should be withheld for at least 3days and in those with a $\mathrm{CrCl}<30 \mathrm{~mL} / \mathrm{min}$ (for which the half-life is $>24 \mathrm{~h}$ ), the drug should be withheld for at least 5 days (10-12).

\section{Treatment of bleeding with dabigatran}

If significant active bleeding occurs, dabigatran should be witheld and supportive care should ensue including transfusing red blood cells and administering intravenous fluids to support haemodynamic instability and promote renal excretion of the drug. Idarucizumab can be prescribed to reverse the effects of dabigatran. Endoscopic therapy for haemostasis of gastrointestinal bleeding may be required. Other therapies that maybe considered in emergencies are prothrombin complex (PCC), haemodialysis, gastric lavage or recombinant activated Factor VIIa. ${ }^{11}$

\section{Rivaroxaban and monitoring of coagulation}

The onset of action for rivaroxaban is 1-3hours. A normal PT excludes a lack of clinically relevant anticoagulation effect. Antifactor Xa levels correlate well with plasma drug concentrations, but may not be readily available in emergency situations. There is no effect on the APTT, TT and ECT., 911

\section{Rivaroxaban and renal function}

Rivaroxaban should be avoided in the presence of advanced liver disease or severe renal impairment. In the presence of normal renal function, the half-life of rivaroxaban is 5 -9hours in young patients and 11-13hours in elderly patients. In patients with normal renal function rivaroxaban should be withheld for 1 to 2 days before highrisk surgical procedures. Recommendations for drug discontinuation prior to surgery are 2days for patients with a $\mathrm{CrCl}$ of $60-90 \mathrm{~mL} / \mathrm{min}$, 5 days for a $\mathrm{CrCl}$ of 30 to $59 \mathrm{~mL} / \mathrm{min}, 7$ days for a $\mathrm{CrCl}$ of $15-29 \mathrm{~mL} /$ min to allow adequate time for complete drug elimination. If the $\mathrm{CrCl}$ is $<15 \mathrm{~mL} / \mathrm{min}$, then elective procedures should be postponed until complete drug metabolism occurs. Rivaroxaban should not be prescribed in patients with stage $\mathrm{V}$ chronic kidney disease.

\section{Treatment of bleeding with rivaroxaban}

In the setting of significant bleeding the same general concepts apply as for dabigatran with supportive care and endoscopic therapy for gastrointestinal bleeding. If life-threatening bleeding occurs PCC can be considered. Dialysis is not effective. ${ }^{11}$

\section{Apixaban and monitoring of coagulation}

The onset of apixaban is 1-3hours, with a half-life of 8-15hours and is less dependent on renal elimination. The mechanism of action is similar to rivaroxaban so similar laboratory coagulation profile is expected. Anti-factor Xa levels correlate well with plasma drug concentrations but may not be readily available in emergency situations. ${ }^{8}$ The APTT, PT, TT, ECT are insensitive and are not useful for assessment of plasma levels of apixaban. ${ }^{9,11}$

\section{Apixaban and renal function}

Apixaban should be discontinued for 1day prior to procedures associated with minimal bleeding and 2 days if there is a high risk of bleeding associated with the surgical procedure providing $\mathrm{CrCl}$ is $>30 \mathrm{ml} / \mathrm{min}$. If $\mathrm{CrCl}$ is $>15-30 \mathrm{ml} / \mathrm{min}$ then discontinuation is required for 3days regardless of the type of surgical procedure. Apixaban is contraindicated in patients with $\mathrm{CrCl}<15 \mathrm{ml} / \mathrm{min}$. ${ }^{11}$

\section{Treatment of bleeding with apixaban}

In the setting of significant bleeding the same general concepts apply as for the other NOACS. Apixaban should be witheld and supportive therapy should commence. Activated charcoal or PCC may be considered in the presence of severe bleeding unresponsive to conservative measures. ${ }^{13}$

\section{Edoxaban}

Edoxaban is the latest NOAC that has been approved for non valvular AF, treatment and prevention of thrombolic events. The onset of action for edoxaban is between 1-2hours and $50 \%$ is excreted via the kidneys. Edoxaban $30 \mathrm{mg}$ and $60 \mathrm{mg}$ OD has been found to be non inferior to warfarin in the prevention of stroke or systemic embolism. There was also significantly lower rates of bleeding with edoxaban. It is contraindicated in patients who have $\mathrm{CrCl}$ of $<30 \mathrm{ml} /$ min. ${ }^{14}$ Since it has been recently introduced there is a lack of data regarding discontinuation for invasive procedures, the effect on laboratory coagulation profile and treatment of bleeding. The PT and APTT maybe prolonged in the presence of edoxaban but this has no relation with bleeding risk. There is no data available with the use of ECT and anti factor Xa levels. The TT cannot be used to assess plasma concentrations. $^{9}$

\section{Specific treatments for bleeding}

Gastric lavage and oral charcoal may be considered depending on the time of the last dose of the NOAC (within eight hours for rivaroxaban, 6hours for apixaban, 2 hours for edoxaban). More than $99.9 \%$ of dabigatran has been found to be absorbed by activated charcoal. No similar effect has been reported for rivaroxaban or apixaban but charcoal maybe considered depending on time of ingestion. Tranexamic acid is an inhibitor of fibinolysis. This should be considered in life threatening bleeding although clinical trial data is lacking. ${ }^{15}$

Haemodialysis/haemofiltration:Dabigatran can be dialysed as there is relatively low ( $\square 35 \%$ ) plasma protein binding. ${ }^{16}$ If an overdose or severe bleeding occurs haemodialysis may be effective in the clearance of dabigatran particularly where more rapid reversal of the anticoagulant effect of is required and in patients with renal impairment. The plasma concentration of dabigatran is reduced by $65 \%$ at $2-4$ hours with haemodialysis. However, haemodialysis is not effective for other NOACs (rivaroxaban, apixaban, and edoxaban) as they are bound to plasma proteins in higher proportions than dabigatran. ${ }^{17,18}$

Recombinant activated factor VIIainitiates haemostasis at sites of bleeding by directly activating thrombin on the surface of platelets. Recombinant activated factor VIIa may potentially reverse the effects of anticoagulants including the NOACs in patients with refractory life-threatening haemorrhage. However the use of this agent in such patients has not been firmly established. 
Prothrombin complex concentrates (PCC) contain all of the vitamin K-dependent coagulation factors and provides rapid reversal of coagulopathy in patients who have been prescribed vitamin $\mathrm{K}$ antagonists. ${ }^{19} \mathrm{PCC}$ can be prescribed for patients taking NOACs with clinically significant bleeding, however there is no clinical evidence for this. ${ }^{10}$ Supporting evidence for the use of PCC and recombinant activated factor VIIa is mostly limited to healthy human volunteers, animal models and in vitro studies.

\section{Antidotes for NOAC}

At present idarucizumab is the approved antidote for significant bleeding with dabigatran. There are no antidotes for rivaroxaban, apixaban and edoxaban. In the RE-VERSE AD trial idarucizumab a monoclonal antibody fragment bound to dabigatran 350 times more than that observed with thrombin. ${ }^{20}$ In patients with normal to mild renal failure idarucizumab produced complete reversal of dabigatran in $88-98 \%$ of patients who had elevated clotting times. Idarucizumab was found to be effective in patients who were prescribed dabigatran and had uncontrollable, life threatening bleeding or in patients who required surgery that could not be delayed for 8hours for which normal coagulation was required. ${ }^{21}$

\section{Conclusion}

The NOACS have been shown to have at least equal efficacy profile in the prevention of stroke in non-valvular $\mathrm{AF}$ and the treatment of venous thromboembolic disease. A major perceived disadvantage however, is the lack of ease in reversibility if there is inadvertent bleeding. The first step in the case of bleeding is to stop the NOAC and support the circulation with intravenous fluids and transfusing red blood cells as required. If this fails other measures include prescribing prothrombin complex, tranexamic acid, activated charcoal and recombinant activated factor VIIa but there is a lack of trial data for these agents. At present only dabigatran has a licensed antidote. Further studies are required to help determine how to manage major bleeding events in patients prescribed NOACS

\section{Acknowledgments}

None.

\section{Conflicts of interest}

Author declares there is no conflicts opf interest.

\section{Funding}

None.

\section{References}

1. Ezekowitz MD, Bridgers SL, James KE, et al. Warfarin in the prevention of stroke associated with nonrheumatic atrial fibrillation. Veterans Affairs Stroke Prevention in Nonrheumatic Atrial Fibrillation Investigators. $N$ Eng J Med. 1992;327(20):1406-1412

2. Connolly SJ, Laupacis A, Gent M, et al. Canadian Atrial Fibrillation Anticoagulation (CAFA) study Journal of the American College of Cardiology. J Am Coll Cardiol. 1991;18(2):349-355.

3. The Boston Area Anticoagulation Trial for Atrial Fibrillation Investigators. The effect of low-dose warfarin on the risk of stroke in patients with non-rheumatic atrial fibrillation. $N$ Eng $J$ Med. 1990;323(22):1505-1511.
4. Granger CB, Alexander JH, McMurray JJ, et al. Apixaban versus warfarin in patients with atrial fibrillation. $N$ Eng J Med . 2011;365(11):981-992.

5. Connolly SJ, Ezekowitz MD, Yusuf S, et al. Dabigatran versus warfarin in patients with atrial fibrillation. $N$ Eng J Med. 2009;361(12):1139-1151.

6. Patel MR, Mahaffey KW, Garg J, et al. Rivaroxaban versus warfarin in nonvalvular atrial fibrillation. $N$ Engl J Med . 2011;365(10):883-891.

7. Hylek EM, Held C, Alexander JH, et al. Major bleeding in patients with atrial fibrillation receiving apixaban or warfarin in the ARISTOTLE trial: predictors, characteristics, and clinical outcomes. J Am Coll cardiol. 2014;63(20):2141-2147.

8. Cuker A, Siegal DM, Crowther MA, et al. Laboratory measurements of anticoagulant activity of the non-vitamin $\mathrm{K}$ oral anticoagulants. $\mathrm{J} \mathrm{Am}$ Coll Cardiol . 2014;64(11):1128-1139.

9. Heidbuchel H, Verhamme P, Alings M, et al. European heart and rhythm association practical guide on the use of new oral anticoagulants in patients with non-valvular atrial fibrillation. Europace . 2013;17(10):625-651.

10. Van Ryn J, Stangier J, Haertter S, et al. Dabigatran etexilate - a novel, reversible, oral direct thrombin inhibitor: interpretation of coagulation assays and reversal of anticoagulant activity. Thrombosis and Haemostasis. 2010;103(6):1116-1127.

11. Baron TH, Kamath PS, McBane, RD. New anticoagulant and antiplatelet agents: a primer for the gastroenterologist. Clin Gastroenterol Hepatol. 2014;12(2):187-195.

12. Stangier J, Rathgen $K$, Stahle $H$, et al. Influence of renal impairment on the pharmacokinetics and pharmacodynamics of oral dabigatranetexilate: an open-label, parallel-group, single-centre study. Clinical Pharmacokinetics. 2010;49(4):259-268.

13. Radaelli F, Dentali F, Repici A, et al. Management of anticoagulation in patients with acute GI bleeding. Dig Liver Dis. 2015;47(8):621-627.

14. Giugliano RP, Ruff CT, Braunwald E, et al. Edoxaban versus warfarin in patients with atrial fibrillation. N Eng J Med . 2013;369(22):2093-2104.

15. Sarich TC, Seltzer JH, Berkowitz SD, et al. Novel oral anticoagulants and reversal agents: Considerations for clinical development. Am Heart J. 2015;169(6):751-757.

16. European Medicines Agency, Pradaxa. Summary of Product Characteristics.

17. Fawole A, Daw HA, Crowther MA. Practical management of bleeding due to the anticoagulants dabigatran, rivaroxaban, and apixaban. Cleveland Clinic Journal of Medicine. 2013;80(7):443-451.

18. Stangier J, Rathgen K, Stähle H, et al. Influence of renal impairment on the pharmacokinetics and pharmacodynamics of oral dabigatran etexilate. Clin Pharmacokinet . 2010;49(4):259-268.

19. Leissinger CA, Blatt PM, Hoots WK, et al. Role of prothrombin complex concentrates in reversing warfarin anticoagulation: a review of the literature. Am J Hematol. 2008; 83(2):137-143.

20. Schiele F, van Ryn J, Canada K, et al. A specific antidote for dabigatran: functional and structural characterization. Blood. 2013;121(18):3554-3562.

21. Pollack CV, Reilly PA, Eikelboom J, et al. Idarucizumab for dabigatran reversal. $N$ Eng J Med. 2015;373(6):511-520. 